\title{
HARQ-Chaotic: Analog Chaotic Code Applied in HARQ Scheme of Wireless Communication System
}

\author{
Wei Yu $\mathbb{D}^{1},{ }^{1,2}$ Fusheng Zhu, ${ }^{3}$ Jun Wu $\mathbb{D}^{1,2}$ Rui Wang, ${ }^{1}$ Haoqi Ren, ${ }^{1}$ and Zhifeng Zhang ${ }^{1}$ \\ ${ }^{1}$ College of Electronic and Information Engineering, Tongji University, Shanghai, China \\ ${ }^{2}$ Key Lab of Ministry of Education in Embedded System and Service Computing, Tongji University, Shanghai, China \\ ${ }^{3}$ GuangDong Communications \& Networks Institute, Guangdong, China \\ Correspondence should be addressed to Jun Wu; wujun@tongji.edu.cn
}

Received 4 January 2019; Revised 10 March 2019; Accepted 19 March 2019; Published 24 April 2019

Academic Editor: Michael McGuire

Copyright (C) 2019 Wei Yu et al. This is an open access article distributed under the Creative Commons Attribution License, which permits unrestricted use, distribution, and reproduction in any medium, provided the original work is properly cited.

\begin{abstract}
This paper proposes a novel symbol-level combining hybrid automatic repeat request (HARQ) scheme based on analog chaotic code and named HARQ-Chaotic. The transmitter of HARQ-Chaotic adopts analog chaotic code to encode Quadrature Amplitude Modulation (QAM) symbols of retransmission packets to combat fading and noise. As the analog chaotic code can only handle sources with amplitudes in the range of [-0.5, 0.5], QAM symbols must be scaled into this range. We derived the optimal scaling factor through theoretical analysis. A joint algorithm combining with novel soft chaotic decoder and novel soft QAM demapper is proposed for the receiver to enhance the performance of the whole communication system. We implemented HARQ-Chaotic with LDPC codes and 16-QAM/64-QAM to carry out simulations in both AWGN channels and multipath fading channels. Massive simulation results demonstrate that the proposed HARQ-Chaotic has $1 d B-4 d B$ gain over traditional HARQ-Chase combining (HARQ-CC) scheme in block error rate (BLER) performance.
\end{abstract}

\section{Introduction}

As wireless channel is time varying and subject to fading, additive noise, and interference [1], hybrid automatic repeat request (HARQ) techniques that combine both forwarderror-correcting (FEC) codes and ARQ are often adopted to improve spectrum efficiency and transmission reliability. Now HARQ has been widely used by many wireless communication systems and standards, such as 3GPP longterm evolution (LTE) [2], IEEE 802.16e WiMAX [3-5], IEEE 802.11 wireless local-area networks (WLANs) [6], etc. There are mainly two types of HARQ, which are HARQ-Chase combining (HARQ-CC) [7] and HARQ incremental redundancy (HARQ-IR) [8]. In HARQ-CC system, the transmitter sends the same source packet repeatedly in retransmission rounds; then the receiver combines retransmission packets in symbol-level according to maximal-ratio combining (MRC) to carry out demodulation and FEC decoding. In HARQ-IR system, the transmitter varies the FEC coding rate and modulation constellation in retransmission packets, which are redundancy bits punctured out from the original transmission packet. The receiver of HARQ-IR combines retransmission packets in bit-level. Previous literatures show that the performance of HARQ-IR is better than HARQ-CC, but the communication system of HARQ-IR is much more complicated [9], so HARQ-CC is more widely used in many wireless communication systems.

As we know the channel fading and noise affect QAM symbols directly, while the FEC coding of HARQ-CC combat fading and noise is indirectly in bit-level. So an intuitive question is whether these QAM symbols can be protected directly in symbol-level. In view of this question, this paper proposes a novel symbol-level HARQ scheme based on analog chaotic code and named HARQ-Chaotic. Many literatures indicate that analog chaotic code has better performance than linear analog codes, especially in the middle and high channel SNR [10-13]. However, previous studies on analog chaotic code only focus on analog-valued sources. This paper extends it to digital-valued sources: the proposed HARQ-Chaotic uses analog chaotic code to encode digital QAM modulation 
symbols of retransmission packets to combat fading and noise directly.

Traditional analog chaotic decoding algorithms work in the way of hard decision, which discard the probability information of the decoding value. If these traditional decoding algorithms are applied to the proposed HARQ-Chaotic system directly, the lost probabilities will reduce the overall system performance. To solve this problem, we propose a soft joint algorithm combining with a novel soft chaotic decoder and a novel soft QAM demapper. The soft chaotic decoder does not make any hard decision but outputs all of the potential decoding values and their corresponding probabilities to the subsequent soft QAM demapper, which outputs log likelihood ratio (LLR) to FEC decoder to recover source bits.

The analog chaotic code can only handle real-valued sources with amplitudes in the range of [-0.5, 0.5]; QAM symbols must be scaled into this range before encoding. How to choose the optimal scaling factor is a trade-off problem. We propose a theoretical method to find the optimal scaling factor by minimizing the noise power of the decoding value after soft chaotic decoder and inverse scaling.

Main contributions of this paper are as follows.

(1) We propose a novel symbol-level HARQ scheme based on analog chaotic code named HARQ-Chaotic, which can protect QAM symbols of retransmission packets to combat fading and noise directly.

(2) We propose a soft joint algorithm combining with a novel soft chaotic decoder and a novel soft QAM demapper, which can improve the performance of the whole communication system greatly.

(3) Through theoretical analysis, we derived the optimal scaling factor to scale QAM symbols into the range of amplitudes that the analog chaotic code can handle.

The organization of the rest of this paper is as follows. Section 2 discusses related works of analog chaotic code and combining schemes of HARQ. Section 3 presents the system model of HARQ-Chaotic. Section 4 describes the detailed design of HARQ-Chaotic, which includes 3 parts. Section 4.1 introduces the analog chaotic encoder and analyses shortcomings of traditional hard decision decoding algorithms. Section 4.2 describes the proposed soft joint algorithm of soft chaotic decoder and soft QAM demapper. Section 4.3 derives the optimal scaling factor through theoretical analysis. We implement HARQ-Chaotic with LDPC code and 16-QAM/64-QAM modulation to carry out simulations in Section 5. Simulation results of HARQ-Chaotic and HARQCC are evaluated and compared under AWGN channels and multipath fading channels. Section VI is the conclusion of this paper.

\section{Related Works}

2.1. Analog Chaotic Code in Wireless Communication Systems. Due to the significant advantage of wideband characteristics provided by chaotic nonlinear signals, a large number of chaos-based communication systems have been proposed exploiting the properties of chaotic waveforms [14], especially in the areas of secure communications [15-17] and spreadspectrum modulation [18-20].

Analog chaotic code is an important application of chaotic signals in wireless communication systems, which is first proposed by Chen and Wornell [12]. The encoder of analog chaotic code treats a single time-discrete, realvalued source symbol as the initial state, and then uses nonlinear chaotic function to generate subsequent states iteratively. As we know, a chaotic system has the butterfly effect phenomenon: two initial states that have very little difference can be totally different in subsequent states. Since the butterfly effect has the same function as the distance expansion in digital coding, therefore, the analog chaotic code uses the initial and subsequent states as codewords to protect the source symbol.

Early research on analog chaotic code focused on efficient decoding algorithms to estimate source symbols from additive white Gaussian noise (AWGN) channel, such as the Maximum-Likelihood (ML) decoder [21], the ExpectationMaximization (EM) algorithm [22], the Bayesian estimation $[23,24]$, etc.

Recently, some new encoding methods based on analog chaotic code have been proposed. For example, Jing $\mathrm{Li}$ proposed Chaotic Analog Turbo (CAT) code [25-27], which works like traditional Turbo codes to explore two parallel but direction-reversed chaotic codeword sequence to strengthen the protection of source symbol. Reference [28] proposed a novel analog chaotic code and applies it to pseudo-analog video wireless communications. Reference [29] proposed a novel hybrid digital-analog code based on analog chaotic code and rateless Spinal code [30], which greatly improves the MSE performance compared with previous pure analog chaotic codes. However, the above papers only apply analog chaotic code to analog (or pseudo-analog) communications systems; this paper extends it to digital communications systems.

2.2. Combining Schemes of HARQ. The combining scheme is very important for the performance of a HARQ system. The proposed soft joint algorithm of this paper is essentially a novel symbol-level combining scheme. We investigated a variety of existing symbol-level combining schemes here. Reference [31] uses a constellation rearrangement with symbol combining to enhance the HARQ performance. The receiver at first combines QAM symbols before demodulation and then demodulates combined symbols with new constellation. Reference [32] investigated three types of combining scheme of HARQ, which are the distance-level combining scheme (DLC), the maximal-ratio combining scheme (MRC), and the bit-level combining scheme (BLC). Simulation results demonstrated that MRC and DLC have the same performance, which are better than BLC. Reference [33] proposed a concatenation-assisted symbol-level combining (CASLC) scheme of HARQ-CC for MIMO systems based on QR decomposition. CASLC scheme has the same performance as MRC scheme but only requires very small memory size. Reference [34] investigated the performance of symbollevel combining (SLC) and BLC schemes of HARQ-CC and gave a conclusion that SLC outperforms BLC in the entire 


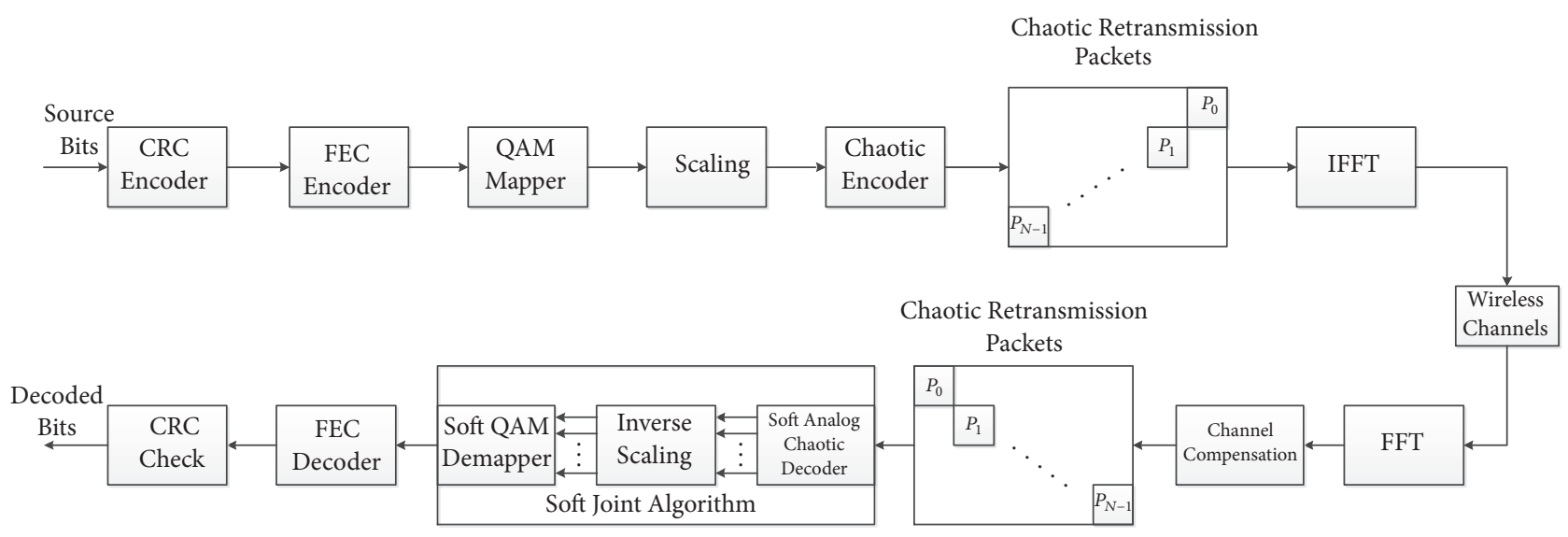

FIGURE 1: Wireless communication system based on HARQ-Chaotic scheme.

SNR region and can even achieve a better throughput than HARQ-IR at the high SNR region.

\section{System Model and Preliminaries}

Figure 1 shows the diagram of the proposed wireless communication system. We use adaptive modulation and coding (AMC) $[35,36]$ to adapt the time-varying channel condition and use the proposed HARQ-Chaotic scheme to enhance the transmission reliability. In AMC scheme, the transmitter chooses the most appropriate PHY mode to match the estimated channel state information (CSI). There are 8 PHY modes with different modulation constellations and FEC coding rates that can be selected by the AMC of IEEE 802.11n, which are listed in Table 1. The IEEE 802.11n standard has two models for FEC codes, which are interleaved convolutional codes (the mandatory model) and low density parity check (LDPC) codes (the optional model). Due to the good performance and wide application of LDPC, we choose it as the FEC codes in this paper.

As we know the higher the modulation constellation that is used, the shorter the expected transmission time will be, but the less likely the delivery will succeed within the frame retry limit [37], so HARQ scheme is often adopted in the case of high order modulation. For this reason, this paper only considers two high order modulation constellations of 16-QAM and 64-QAM, that is, mode 4 to mode 8 of Table 1. As too many retransmissions can cause long system delay and low spectrum efficiency, many realistic wireless communication systems adopt limited number of retransmission rounds, which is called truncated HARQ (THARQ). In this paper, we only consider the number of retransmission rounds to be 2 and 3 .

Orthogonal frequency division multiplexing (OFDM) divides the whole available bandwidth into multiple orthogonal subcarriers, which is well suited to broadband systems in frequency selective fading environments. For this reason, OFDM is adopted in our system. There are total of 52 subcarriers: 48 of them carry actual QAM modulation symbols and the rest 4 subcarriers carry pilots which are used for phase tracking and channel estimation. The realization of OFDM is
TABLE 1: 8 PHY modes of IEEE 802.11.

\begin{tabular}{lcc}
\hline Mode & Modulation & Code Rate \\
\hline 1 & BPSK & $1 / 2$ \\
2 & QPSK & $1 / 2$ \\
3 & QPSK & $3 / 4$ \\
4 & 16-QAM & $1 / 2$ \\
5 & 16-QAM & $3 / 4$ \\
6 & 64-QAM & $2 / 3$ \\
7 & 64-QAM & $3 / 4$ \\
8 & 64-QAM & $5 / 6$ \\
\hline
\end{tabular}

Inverse Fast Fourier Transformation (IFFT) at the transmitter and Fast Fourier Transformation (FFT) at the receiver.

The entire data flow is described as follows. The Cyclic Redundancy Check (CRC) module generates CRC check bits and added them at the tail of the source bits packet. FEC encoder and QAM mapper work as the traditional way to encode and modulate source bits into QAM symbols. Then analog chaotic encoder encodes each plane (I or Q plane) of scaled QAM symbols to real-valued chaotic codewords. (the analog chaotic code in this paper can be regarded as a kind of systematic rateless codes, which can generate infinite number of codewords.) Two real-valued chaotic codewords corresponding to a QAM symbol are combined into one complex-valued chaotic codeword. Suppose a scaled QAM symbol is $a+b \cdot j$, the coding rate of analog chaotic code is $1 / N$. Then real-valued codewords of $\{c[0], c[1], \ldots, c[N-1]\}$ and $\{d[0], d[1], \ldots, d[N-1]\}$ are generated from the I and Q plane, respectively. After combining, we get complex-valued codewords of $\{e[0], e[1], \ldots, e[N-1]\}$ as follows:

$$
\begin{gathered}
e[0]=c[0]+d[0] \cdot j=a+b \cdot j, \\
e[1]=c[1]+d[1] \cdot j, \\
\vdots \\
e[N-1]=c[N-1]+d[N-1] \cdot j .
\end{gathered}
$$


Then $e[0]$ is allocated to the original transmission packet of $P_{0}, e[1]$ is allocated to the first retransmission packet of $P_{1}, \ldots, e[N-1]$ is allocated to the $(N-1)$ th retransmission packet of $P_{N-1}$. IFFT transforms these chaotic retransmission packets from frequency domain to time domain and outputs them to the wireless channel. After sending a packet, the transmitter waits for the feedback (ACK/NAK) from the receiver. When the receiver receives a packet, it transforms the packet from time domain to frequency domain using FFT at first and then tries to recover it. The recovery process includes channel compensation, soft chaotic decoding, inverse scaling, soft QAM demapping, and FEC decoding. If the packet is successfully recovered (CRC checking is correct), the receiver sends a HARQ ACK signal to the transmitter; otherwise a HARQ NAK signal is sent.

The recovery process at the receiver is divided into two cases according to whether the packet is an original transmission packet or a retransmission HARQ-Chaotic packet. Case 1: the packet is an original transmission packet. The QAM demapper and the FEC decoder work in the traditional way to process this packet in sequence. If the source bits cannot be decoded correctly, this packet is saved for the combining of next retransmission rounds. Case 2: the packet is a retransmission packet with analog chaotic encoding. The soft chaotic decoder combines all of the retransmission packets and the original transmission packet together to output all of the potential decoding values and their corresponding probabilities. Then the soft QAM demapper processes the inverse scaled decoding values and their corresponding probabilities to output LLRs to the FEC decoder. If the source bits cannot be decoded correctly, this packet is also saved for the combining of next retransmission rounds.

\section{The Design of HARQ-Chaotic}

4.1. The Analog Chaotic Encoder and the Analysis of Traditional Hard Decision Decoding Algorithms. Chen et al. [12] first proposed analog chaotic code based on tent map function. Other papers inspired by [12], such as $[13,25,27$, 29 ], also use tent map function to design and analyze new analog chaotic codes. Due to the good performance and extensive use of tent map function, we also use it in this paper.

A rate $1 / N$ analog chaotic code takes in a single source symbol $u(u \in R,-0.5 \leq u \leq 0.5)$ at one time and then encodes it to $N$ real-valued chaotic codewords, which are $\{x[0], x[1], \ldots, x[N-1]\})[13]$ where $x[0]=u, x[n]$ and its sign $s[n]( \pm 1)$ are generated from the previous codeword $x[n-1]$ using the tent map function $F($.$) , that is,$

$$
\begin{aligned}
& x[n]=F(x[n-1]), \\
& s[n]=\operatorname{sgn}(x[n]) .
\end{aligned}
$$

where $F($.$) is defined as$

$$
F(x)= \begin{cases}2 x+0.5, & -0.5 \leq x \leq 0 \\ -2 x+0.5, & 0<x \leq 0.5\end{cases}
$$

The inverse ten map function $F_{s}^{-1}($.$) is defined as$

$$
x[n-1]=F_{s[n-1]}^{-1}(x[n])=\frac{0.5-x[n]}{2} \cdot s[n-1] .
$$

After AWGN channel, the receiver gets $N$ chaotic codewords with noise, which are $\{y[0], y[1], \ldots, y[N-1]\}$, i.e.,

$$
y[i]=x[i]+z, \quad i=0,1, \ldots, N-1,
$$

where $z$ is zero mean additive white Gaussian noise with variance $\sigma^{2}$.

Traditional hard decision decoding algorithms estimate $u$ from $\{y[0], y[1], \ldots, y[N-1]\}[21,25]$. Key steps are as follows.

(1) We define $\tilde{x}[n \mid n]$ as the estimation of $x[n]$ from $\overrightarrow{\mathbf{y}}^{n}=$ $\{y[0], y[1], \ldots, y[n]\}$, that is,

$$
\begin{aligned}
\tilde{x} & {[n \mid n]=\underset{-0.5 \leq \tilde{x}[n \mid n] \leq 0.5}{\arg \min } P\left(\overrightarrow{\mathbf{y}}^{n} \mid \tilde{x}[n \mid n]\right) } \\
& =\underset{-0.5 \leq \tilde{x}[n \mid n] \leq 0.5}{\arg \min } \sum_{k=0}^{n}(y[k] \\
& \left.-F_{s[k], s[k+1], \ldots, s[n-1]}^{(k-n)}(\tilde{x}[n \mid n])\right)^{2} \\
& =\underset{-0.5 \leq \tilde{x}[n \mid n] \leq 0.5}{\arg \min } \sum_{k=0}^{n} 2^{2(k-n)}\left(F^{(n-k)}(y[k])\right. \\
& -\tilde{x}[n \mid n])^{2},
\end{aligned}
$$

where $F^{k}($.$) and F_{s_{0}, s_{1}, \ldots, s_{k-1}}^{-k}($.$) are defined as$

$$
\begin{aligned}
F^{k}(x) & =\underbrace{F(F(\cdots(F}_{k}(x)))), \\
F_{s_{0}, s_{1}, \ldots, s_{k-1}}^{-k}(x) & =\underbrace{F_{s_{0}}^{-1}\left(F _ { s _ { 1 } } ^ { - 1 } \left(\cdots \left(F_{s_{k-1}}^{-1}\right.\right.\right.}_{k}(x)))) .
\end{aligned}
$$

(2) By differentiating the sum item of (6) with respect to $\tilde{x}[n \mid n]$ and letting the derivative equal to 0 , we can get $\tilde{x}[n \mid$ $n]$ and its sign as follows:

$$
\begin{aligned}
\tilde{x}[n \mid n] & =\frac{\sum_{k=0}^{n} 2^{2(k-n)} F^{(n-k)}(y[k])}{\sum_{k=0}^{n} 2^{2(k-n)}}, \\
\tilde{s}[n] & =\operatorname{sgn}(\tilde{x}[n \mid n]) .
\end{aligned}
$$

(3) $\tilde{x}[N-1 \mid N-1]$ and $\{\widetilde{s}[0], \widetilde{s}[1], \ldots, \widetilde{s}[N-2]\}$ can be obtained from the above equation directly; then the source symbol $x[0]($ or $u)$ can be decoded as

$$
\begin{aligned}
\widetilde{u} & =\tilde{x}[0 \mid N-1] \\
& =F_{\widetilde{s}[0], \widetilde{s}[1], \ldots, \widetilde{s}[N-2]}^{-(N-2)}(\widetilde{x}[N-1 \mid N-1]) .
\end{aligned}
$$

As we can see, the recovery of signs $\{\widetilde{s}[0], \widetilde{s}[1], \ldots, \widetilde{s}[N-$ $2]\}$ is a key point in the last step. In fact, these signs represent the quantization interval that $u$ belongs to. Suppose the value 


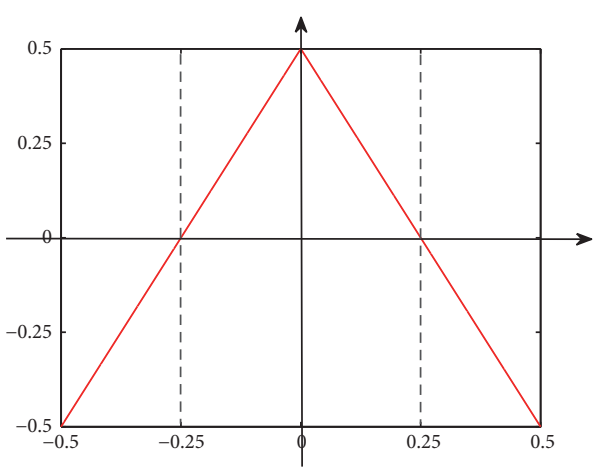

(a) Curve of $x$ [1] (vertical axis) as a function of $u$ (horizontal axis)

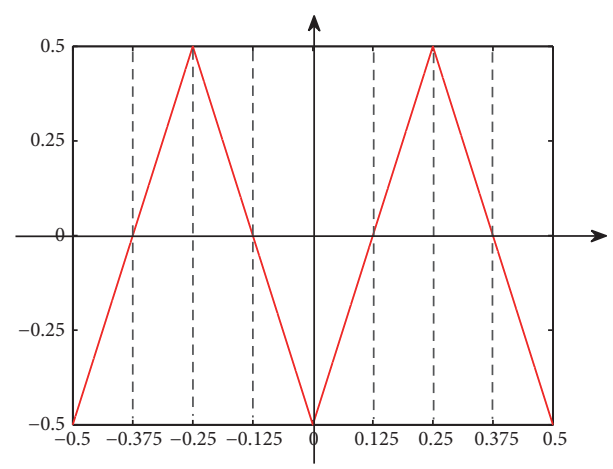

(b) Curve of $x$ [2] (vertical axis) as a function of $u$ (horizontal axis)

FIgURE 2: Tent map functions.

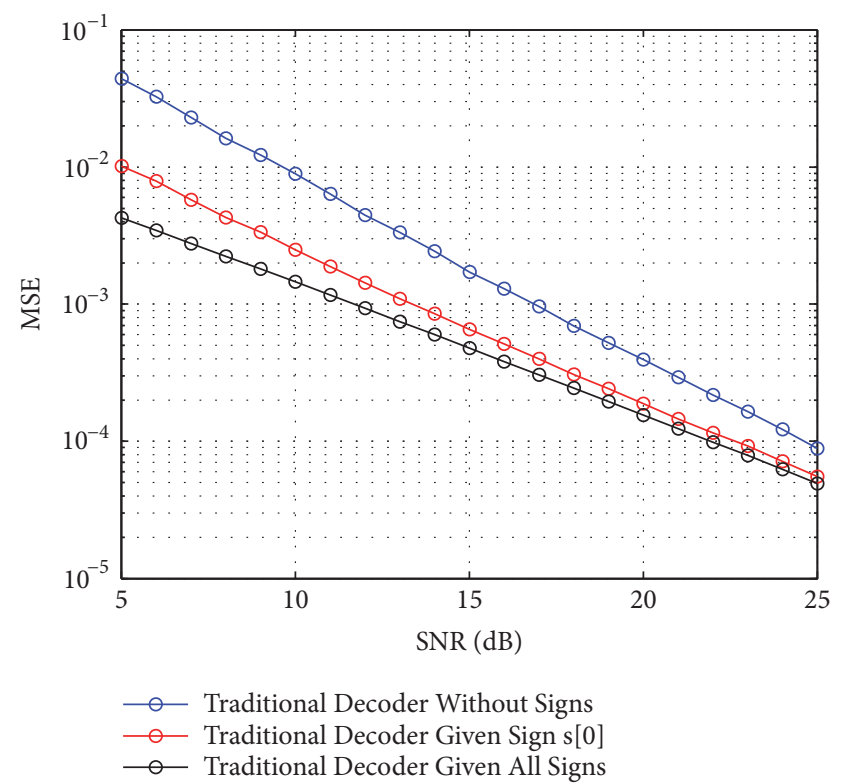

FIGURE 3: MSE performance comparison with and without knowing signs.

of $u$ is -0.15 ; we can get chaotic codewords and signs as $\{x[0]=-0.15, x[1]=0.2, x[2]=0.1, s[0]=-1, s[1]=1$, $s[2]=1\}$. Figures $2(\mathrm{a})$ and $2(\mathrm{~b})$ show curves of $x[1], x[2]$ as a function of $u$. From these two figures, we can get the following information. (1) $s[0]$ determines that $u$ belongs to the interval of $[-0.5,0]$. (2) $s[0]$ and $s[1]$ determine that $u$ belongs to the interval of $[-0.25,0]$. (3) $s[0], s[1]$, and $s[2]$ determine that $u$ belongs to the interval of $[-0.25,-0.125]$, etc. [29].

Figure 3 illustrates the importance of these signs for the decoding mean square error (MSE) performance of analog chaotic code where the channel is AWGN and the coding rate is $1 / 3$. As we can see, a very low decoding MSE can be obtained if the decoder knows all signs correctly. Even only one sign $s[0]$ is given, the decoding MSE can also be highly reduced. Unfortunately, these signs cannot be recovered efficiently in practical systems, and false signs will misjudge the interval of $u$, thereby reducing the decoding performance of analog chaotic code, and then reduce the performance of the overall HARQ-Chaotic system.

\subsection{The Soft Joint Algorithm of Soft Chaotic Decoder and Soft QAM Demapper}

4.2.1. The Novel Soft Chaotic Decoder. In view of the above shortcoming of traditional analog chaotic decoding algorithms, we propose a soft joint algorithm combining a novel soft chaotic decoder and a novel soft QAM demapper. The soft chaotic decoder does not estimate signs or determine the interval that $u$ locates in but outputs all decoding values of $u$ in every interval and their corresponding probabilities. The mission of the proposed soft chaotic decoder is to maximize the probability of $P(x[0], x[1], \ldots, x[N-$ $1] \mid y[0], y[1], \ldots, y[N-1])$, i.e.,

$$
\begin{aligned}
P & (x[0], x[1], \ldots, x[N-1] \mid y[0], y[1], \ldots, y[N-1]) \\
& =\frac{1}{(\sqrt{2 \pi} \sigma)^{N}} \times e^{-\sum_{i=0}^{N-1}(y[i]-x[i])^{2} / 2 \sigma^{2}} .
\end{aligned}
$$

As this paper only considers 2 and 3 retransmission rounds that means $N=2,3$. When $N=2$, the above equation can be rewritten as

$$
P(x[0], x[1] \mid y[0], y[1])= \begin{cases}\frac{1}{(\sqrt{2 \pi} \sigma)^{2}} e^{-\left((y[0]-x[0])^{2}+(y[1]-(2 x[0]+0.5))^{2}\right) / 2 \sigma^{2}}, & -0.5 \leq x[0] \leq 0 \\ \frac{1}{(\sqrt{2 \pi} \sigma)^{2}} e^{-\left((y[0]-x[0])^{2}+(y[1]-(-2 x[0]+0.5))^{2}\right) / 2 \sigma^{2}}, & 0<x[0] \leq 0.5 .\end{cases}
$$


By differentiating the above equation with respect to $x[0]$ and letting the derivative equal to 0 , we can get two possible decoding values of $x[0]$, which are

$$
\begin{array}{ll}
\widetilde{x}_{1}[0]=\frac{y[0]+2 y[1]-1}{5}, & -0.5 \leq \widetilde{x}_{1}[0] \leq 0 \\
\widetilde{x}_{2}[0]=\frac{y[0]-2 y[1]+1}{5}, & 0<\widetilde{x}_{2}[0] \leq 0.5 .
\end{array}
$$

Applying these two decoding values to (11), we can get their corresponding probabilities, i.e., $P_{x 1}$ and $P_{x 2}$.

When $N=3$, we can get 4 possible decoding values of $x[0]$, which are

$$
\begin{aligned}
& \tilde{x}_{1}[0]=\frac{y[0]+2 y[1]+4 y[2]-7}{21}, \\
& -0.5 \leq \tilde{x}_{1}[0]<-0.25 \\
& \tilde{x}_{2}[0]=\frac{y[0]+2 y[1]-4 y[2]-3}{21}, \\
& \tilde{x}_{3}[0]=\frac{y[0]-2 y[1]+4 y[2]+3}{21}, \quad 0.25 \leq \tilde{x}_{2}[0]<0 \\
& \tilde{x}_{4}[0]=\frac{y[0]-2 y[1]-4 y[2]+7}{21}, \quad 0.25 \leq \tilde{x}_{3}[0]<0.25 \\
& 0.0 .5
\end{aligned}
$$

Similarly, we can get the 4 corresponding probabilities of $P_{x 1}, P_{x 2}, P_{x 3}$, and $P_{x 4}$ when applying these 4 decoding values to $(10)$.

The soft chaotic decoder outputs all these possible decoding values of $\tilde{x}_{i}[0]$ and their probabilities $P_{x i}(i=$ $\left.1,2, \ldots,(N-1)^{2}\right)$, which will be processed by the soft QAM demapper.

4.2.2. The Novel Soft QAM Demapper. We take the situation of 16-QAM modulation and 2 retransmission rounds as an example. Figure 4 shows the soft demapping process of the first bit of one 16-QAM symbol. The 16 black spots are constellation positions. We use $\left\{d_{1}, d_{2}, \ldots, d_{16}\right\}$ to denote the complex values of them. Since each plane (I or Q) of one QAM symbol has 2 potential real-valued decoding values, the soft chaotic decoder outputs 4 complex-valued potential decoding values (denoted by $\left\{\widetilde{m}_{1}, \widetilde{m}_{2}, \widetilde{m}_{3}, \widetilde{m}_{4}\right\}$ ) and their corresponding probabilities (denoted by $\left\{P_{m 1}, P_{m 2}, P_{m 3}, P_{m 4}\right\}$, respectively), which are represented by squares in Figure 4 . Meanwhile, we use $\left\{P_{m 1 j}, P_{m 2 j}, P_{m 3 j}, P_{m 4 j}\right\}(i=1,2, \ldots, 16)$ to denote probabilities of sending the $i$ th constellation point and receiving $\widetilde{m}_{1}, \widetilde{m}_{2}, \widetilde{m}_{3}, \widetilde{m}_{4}$ at the receiver, respectively. The 8 black spots on the right side indicate that the first bit is 1 . In contrast, the 8 black spots on the left side indicate that the first bit is 0 . We use $P_{1_{-1}}$ and $P_{1_{-} 0}$ to denote probabilities of the

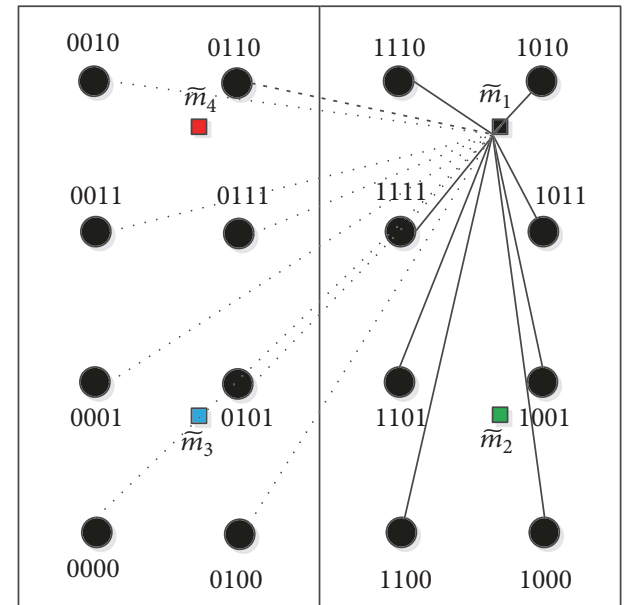

FIGURE 4: Diagram of the soft demapping process of 16-QAM symbol and 2 retransmission rounds.

first bit equal to 1 and 0 , respectively. The calculation of $P_{1_{-}}$ is as follows:

$$
\begin{aligned}
P_{1_{\_} 1}= & P_{m 1} \times \sum_{i=1}^{8} P_{m 1 \_i}+P_{m 2} \times \sum_{i=1}^{8} P_{m 2_{-} i}+P_{m 3} \times \sum_{i=1}^{8} P_{m 3 i} \\
& +P_{m 4} \times \sum_{i=1}^{8} P_{m 4 j} \\
= & P_{m 1} \frac{1}{\sqrt{2 \pi} \sigma_{1}} \sum_{i=1}^{8} \exp \left(-\frac{1}{2 \sigma_{1}^{2}}\left(d_{i}-\widetilde{m}_{1}\right)^{2}\right) \\
& +P_{m 2} \frac{1}{\sqrt{2 \pi} \sigma_{2}} \sum_{i=1}^{8} \exp \left(-\frac{1}{2 \sigma_{2}^{2}}\left(d_{i}-\widetilde{m}_{2}\right)^{2}\right) \\
& +P_{m 3} \frac{1}{\sqrt{2 \pi} \sigma_{3}} \sum_{i=1}^{8} \exp \left(-\frac{1}{2 \sigma_{3}^{2}}\left(d_{i}-\widetilde{m}_{3}\right)^{2}\right) \\
& +P_{m 4} \frac{1}{\sqrt{2 \pi} \sigma_{4}} \sum_{i=1}^{8} \exp \left(-\frac{1}{2 \sigma_{4}^{2}}\left(d_{i}-\widetilde{m}_{4}\right)^{2}\right) .
\end{aligned}
$$

As we can see, $P_{1_{-1}}$ consists of 4 items added together. Each item represents one of the complex-valued decoding values output from the soft chaotic decoder. Let us take the first item as an example to illustrate. $\sum_{i=1}^{8} P_{m 1 i}$ denotes the sum of 8 probabilities that the transmitter sends the 8 constellation points on the right side of Figure 4 and the receiver receives $\widetilde{m}_{1}$. Each transmitted constellation point and the received $\widetilde{m}_{1}$ are connected with a solid line in Figure 4 . The noise power additional to $\left\{\widetilde{m}_{1}, \widetilde{m}_{2}, \widetilde{m}_{3}, \widetilde{m}_{4}\right\}$ is denoted by $\left\{\sigma_{1}, \sigma_{2}, \sigma_{3}, \sigma_{4}\right\}$, respectively, which can be obtained through pilots.

Similarly, we can write $P_{1_{-} 0}$ as follows:

$$
\begin{aligned}
P_{1 \_0}= & P_{m 1} \times \sum_{i=9}^{16} P_{m 1_{\_} i}+P_{m 2} \times \sum_{i=9}^{16} P_{m 2 \_}+P_{m 3} \times \sum_{i=9}^{16} P_{m 3 i} \\
& +P_{m 4} \times \sum_{i=9}^{16} P_{m 4 i}
\end{aligned}
$$




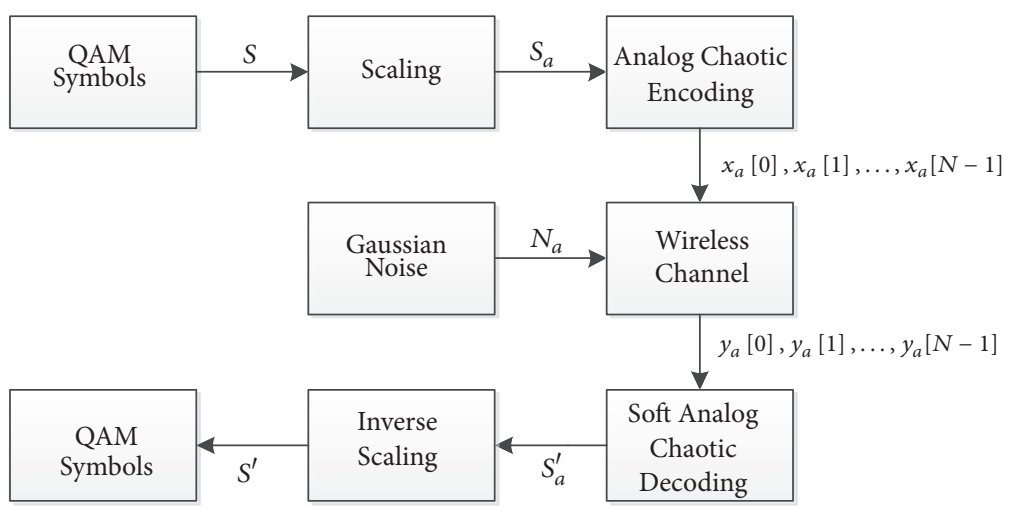

FIGURE 5: The diagram of the simple communication system.

$$
\begin{aligned}
= & P_{m 1} \frac{1}{\sqrt{2 \pi} \sigma_{1}} \sum_{i=9}^{16} \exp \left(-\frac{1}{2 \sigma_{1}^{2}}\left(d_{i}-\widetilde{m}_{1}\right)^{2}\right) \\
& +P_{m 2} \frac{1}{\sqrt{2 \pi} \sigma_{2}} \sum_{i=9}^{16} \exp \left(-\frac{1}{2 \sigma_{2}^{2}}\left(d_{i}-\widetilde{m}_{2}\right)^{2}\right) \\
& +P_{m 3} \frac{1}{\sqrt{2 \pi} \sigma_{3}} \sum_{i=9}^{16} \exp \left(-\frac{1}{2 \sigma_{3}^{2}}\left(d_{i}-\widetilde{m}_{3}\right)^{2}\right) \\
& +P_{m 4} \frac{1}{\sqrt{2 \pi} \sigma_{4}} \sum_{i=9}^{16} \exp \left(-\frac{1}{2 \sigma_{4}^{2}}\left(d_{i}-\widetilde{m}_{4}\right)^{2}\right) .
\end{aligned}
$$

The LLR of the first bit can be obtained as

$$
\operatorname{LLR}\left(b_{1}\right)=\log \left(\frac{P_{1 \_1}}{P_{1 \_0}}\right) .
$$

The LLRs of other 3 bits of one 16-QAM symbol can be calculated in a similar method.

4.3. The Optimal Scaling Factor. In order to derive the optimal scaling factor, we consider a simple communication system shown as Figure 5. The wireless channel is AWGN. Sources to be transmitted are one plane (I or Q) of 16-QAM/64-QAM symbols. We set the 4 unique amplitudes of one plane of 16-QAM symbols to be $\{-3,-1,1,3\}$ and the 8 unique amplitudes of one plane of 64 QAM symbols to be $\{-7,-5,-3,-1,1,3,5,7\}$. As mentioned before, we have to scale these amplitudes into the range of $[-0.5,0.5]$ before analog chaotic encoding. It is very clear that any scaling factor larger than $6 / 14$ can scale $\{-3,-1,1,3\} /\{-7,-5,-3,-1,1,3,5,7\}$ into this range. But how to choose the most appropriate scaling factor is a tradeoff problem. As mentioned before, the soft chaotic decoder outputs all potential decoding values, where, only one value locates in the correct interval. Our solution is to find the scaling factor that minimizes the noise power of the decoding value in the correct interval after soft chaotic decoder and inverse scaling.
We take the situation of 2 retransmission rounds (the coding rate of analog chaotic code is $1 / 2$, and one plane of QAM symbol generates 2 chaotic codewords.) and 16QAM modulation as an example to carry out the following analysis. Table 2 lists notations that will be used. Suppose there are 16 QAM symbols that need to be transmitted; each symbol is a unique position of the 16-QAM constellation. After scaling, the 4 unique amplitudes of each plane are $\{-3 / a,-1 / a, 1 / a, 3 / a\}$. After chaotic encoding, we get 4 pairs of unique real-valued chaotic codewords (totally 8 real-valued chaotic codewords) of one plane, which are $\{-3 / a,-6 / a+1 / 2\},\{-1 / a,-2 / a+1 / 2\},\{1 / a,-2 / a+1 / 2\}$, $\{3 / a,-6 / a+1 / 2\}$. The average power of these 8 real-valued chaotic codewords is

$$
P_{a}=\frac{25}{2 a^{2}}-\frac{2}{a}+\frac{1}{8}
$$

Suppose $S$ is an arbitrary value of $\{-3,-1,1,3\}$. After scaling and analog chaotic encoding, the transmitter sends real-valued chaotic codewords of $\left\{x_{a}[0], x_{a}[1], \ldots, x_{a}[N-1]\right\}$ to the AWGN channel. Then the receiver gets them with noise as

$$
y_{a}[i]=x_{a}[i]+N_{a}, \quad i=0,1, \ldots, N-1,
$$

where the average power of $x_{a}[i]$ and $N_{a}$ are $P_{a}$ and $P_{n_{-}}$, respectively.

After soft chaotic decoder, the decoding value in the correct interval is

$$
S_{a}^{\prime}=S_{a}+N_{a}^{\prime}
$$

Lemma 1. After soft analog chaotic decoding, the average power of noise $N_{a}^{\prime}$ is reduced to be

$$
P_{n \_}^{\prime}=\frac{P_{n\lrcorner a}}{5}
$$

and the proof is shown in the appendix.

After inverse scaling, the receiver gets output in the correct interval as

$$
S^{\prime}=S_{a}^{\prime} \times a=S+N_{a}^{\prime} \times a=S+N^{\prime}
$$


TABLE 2: Summary of notations.

\begin{tabular}{lc}
\hline Notation & Meaning \\
\hline$a$ & scaling factor \\
$S$ & one plane of QAM symbol before scaling \\
$S_{a}$ & after scaling of $S$ \\
$x_{a}[0], \ldots, x_{a}[N-1]$ & real-valued chaotic codewords \\
$P_{a}$ & the SNR of AWGN channel \\
$S N R$ & Gaussian noise of AWGN channel \\
$N_{a}$ & average power of $N_{a}, P_{n a}=P_{a} / S N R$ \\
$P_{n a}$ & received chaotic codewords after AWGN channel \\
$y_{a}[0], \ldots, y_{a}[N-1]$ & decoding value in the correct interval after soft chaotic decoder \\
$S_{a}^{\prime}$ & decoding value in the correct interval after inverse scaling \\
$S^{\prime}$ & additive noise of $S_{a}^{\prime}$ \\
$N_{a}^{\prime}$ & average power of $N_{a}^{\prime}$ \\
$P_{n a}^{\prime}$ & additional noise on $S^{\prime}$ \\
$N^{\prime}$ & average power of $N^{\prime}$ \\
$P_{n}^{\prime}$ & and
\end{tabular}

The average power of noise $N^{\prime}$ is

$$
\begin{aligned}
P_{n}^{\prime} & =P_{n a}^{\prime} \times a^{2}=\frac{P_{n \_a} \times a^{2}}{5}=\frac{P_{a} \times a^{2}}{5 \times S N R} \\
& =\frac{0.125 \times a^{2}-2 \times a+12.5}{5 \times S N R}
\end{aligned}
$$

In order to get the minimum value of $P_{n}^{\prime}$, we differentiate the above equation respective to $a$ and let the derivative equal to 0 . Then we get the optimal scaling factor, that is, $a=8$.

Similarly, we give other optimal scaling factors directly as follows. In the condition of 3 retransmission rounds of 16-QAM modulation, the optimal scaling factor is also 8 . In the condition of 2 and 3 retransmission rounds of 64QAM modulation, both of the optimal scaling factors are 16.

\section{Performance Evaluation}

We evaluated the block error rate (BLER) performance of the proposed HARQ-Chaotic scheme and the traditional HARQ-CC scheme over AWGN channel and IEEE 802.11 fading channel model A. The latter channel is a typical office environment for the Non-Line-of-Sight (NLOS) multiple paths scenario; the detailed specifications can be found in $[38,39]$. The multipath has ten taps, the the root-meansquare (rms) delay spread is set to $90 \mathrm{~ns}$. As mentioned before we consider 2 and 3 retransmission rounds for mode 4 to mode 8 of IEEE 802.11n PHY, that is, coding rates of $1 / 2$, $3 / 4$ for 16-QAM and coding rate of $2 / 3,3 / 4,5 / 6$ for 64 QAM. The number of LDPC coded bits of one packet is set to be 900 for 16-QAM and 1200 for 64-QAM. For every integer SNR, the transmitter sends 8000 data packets. The

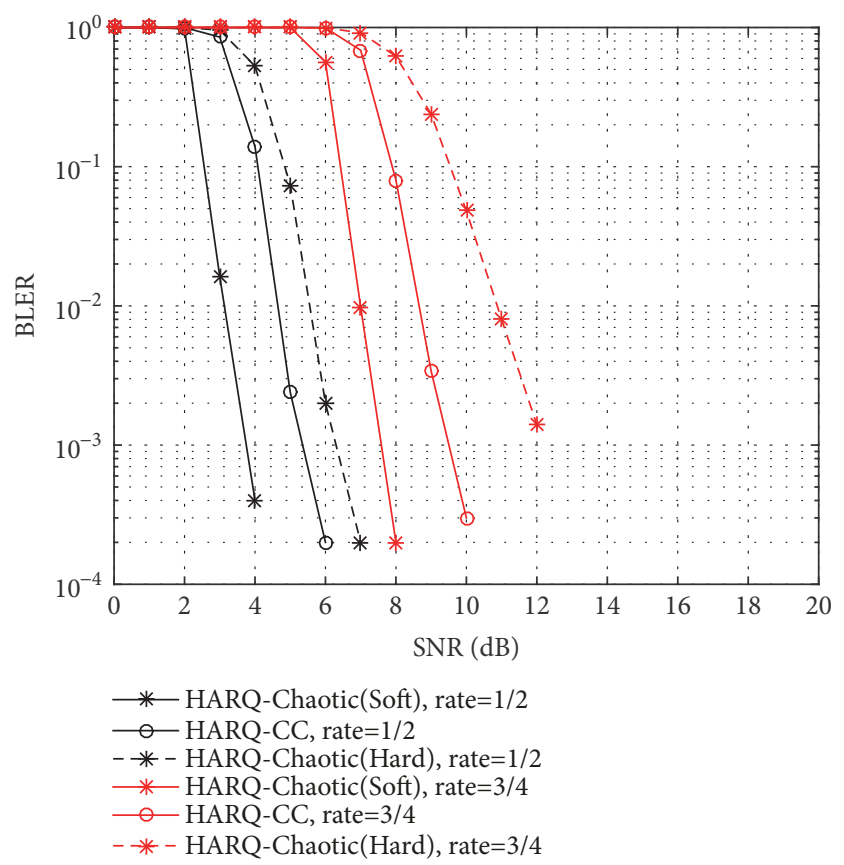

FIGURE 6: BLER of 2 retransmissions with LDPC coding and 16QAM modulation in AWGN channels.

channel equalization is implemented in frequency domain; the number of coefficients of equalization filter is equal to the FFT size, that is, 64 .

Figures 6-9 show the BLER performance comparison in 16-QAM modulation (the curves labeled as "HARQ-Chaotic" in Figures 7-13 represent HARQ schemes based on soft joint algorithm). There are three different HARQ schemes in Figure 6, which are (1) HARQ-Chaotic scheme based on 


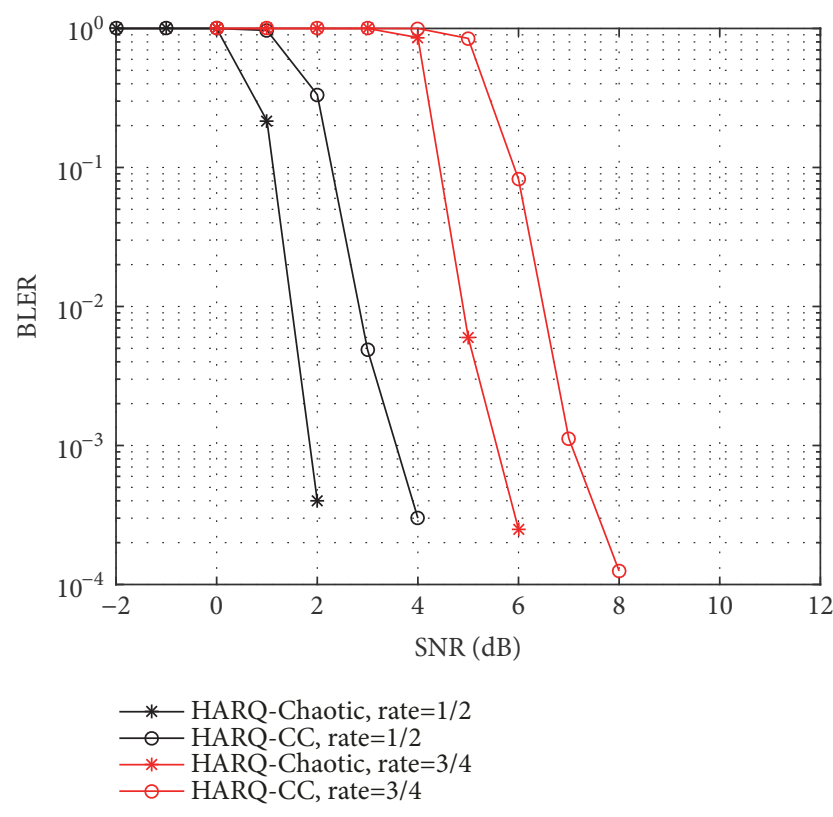

FIGURE 7: BLER of 3 retransmissions with LDPC coding and 16QAM modulation in AWGN channels.

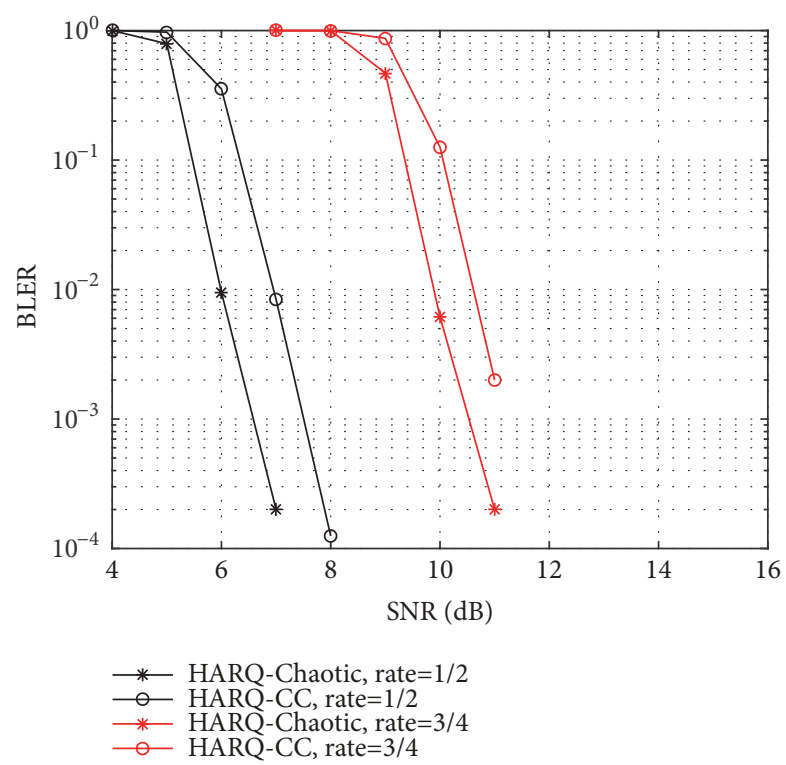

FIGURE 8: BLER of 2 retransmissions with LDPC coding and 16QAM modulation in fading channels.

the traditional hard decision chaotic decoder (indicated by "HARQ-Chaotic (hard)"), (2) HARQ-Chaotic scheme based on the proposed soft joint algorithm (indicated by "HARQChaotic (soft)") and (3) the traditional HARQ-CC scheme (indicated by "HARQ-CC"). As we can see, "HARQ-Chaotic (Soft)" has a huge gain compared with "HARQ-Chaotic (Hard)", which proves the effectiveness of the proposed soft joint algorithm. From Figures 6-9 we can also see that

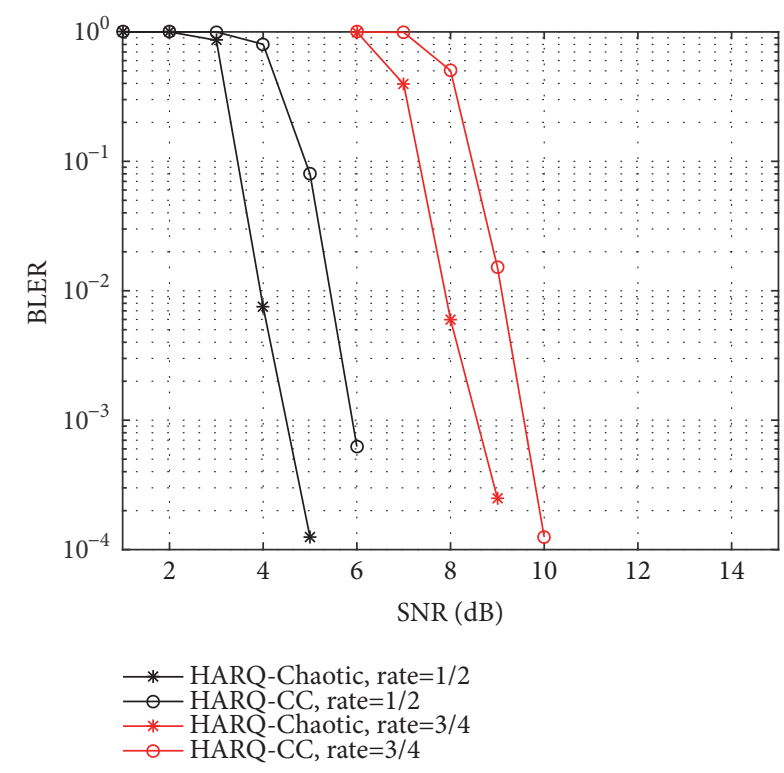

FIGURE 9: BLER of 3 retransmissions with LDPC coding and 16QAM modulation in fading channels.

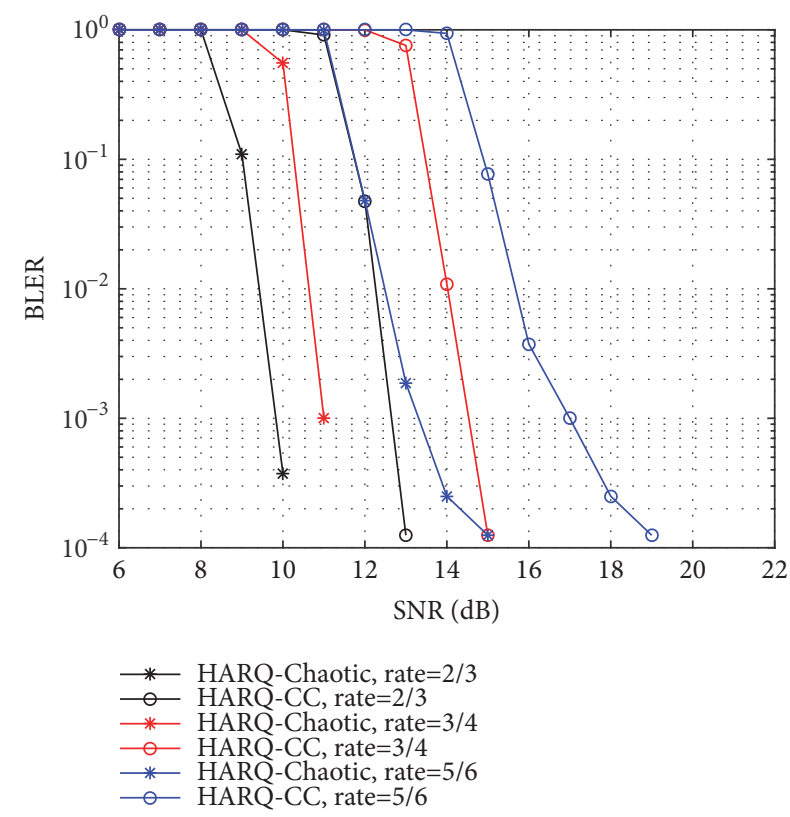

FIGURE 10: BLER of 2 retransmissions with LDPC coding and 64QAM modulation in AWGN channels.

regardless of what kind of scenarios, the proposed HARQChaotic scheme can always have $1-2 d B$ gain comparing with traditional HARQ-CC scheme. Figures 10-13 show the BLER performance comparison in 64-QAM modulation. We can see that when the coding rate is $5 / 6$, the gain is about $2 d B$. When the coding rate is $2 / 3$ and $3 / 4$, the gain can be as large as $3 d B$. The above massive simulation results under different scenarios (different LDPC coding rate, different modulation constellations, different retransmission rounds, and different 


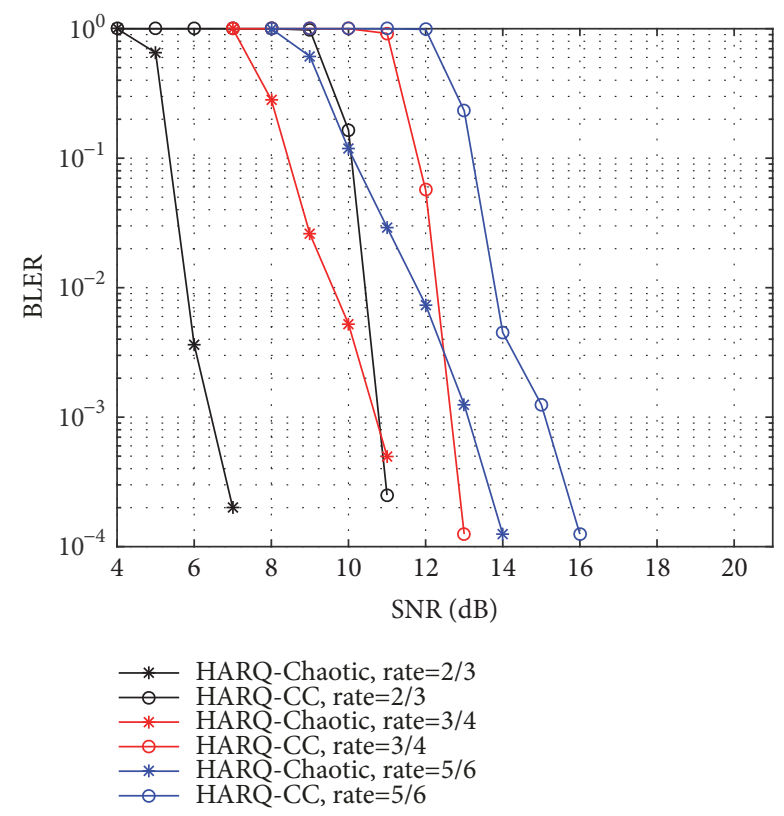

FIGURE 11: BLER of 3 retransmissions with LDPC coding and 64QAM modulation in AWGN channels.

wireless channels) demonstrate that the proposed HARQChaotic scheme has a stable performance gain comparing with HARQ-CC scheme. The gain comes from the following factors. HARQ-CC is equivalent to using repeated linear code to protect QAM symbols, while HARQ-Chaotic uses nonlinear chaotic code. A large number of previous studies [10-13] have shown that analog chaotic code can bring obvious gain compared with linear codes in many scenarios, especially for analog-valued source. When the sources of analog chaotic code are digital QAM symbols, the traditional hard decision chaotic decoding algorithm does not work very well. However, the proposed soft joint algorithm can solve this problem perfectly, which makes the analog chaotic code very suitable for QAM symbols.

\section{Conclusion}

This paper proposes a novel symbol-level HARQ scheme, HARQ-Chaotic based on analog chaotic code. In the past research works, the analog chaotic decoding algorithm is all hard decision, which discards the probability information of the decoding output. The lost probabilities will reduce the performance of the HARQ-Chaotic communication system. We proposed a soft joint algorithm combining with a novel soft chaotic decoder and a novel soft QAM demapper to solve this problem. An optimal scaling factor is derived to scale QAM symbols into the amplitude range that analog chaotic code can handle. We implemented HARQ-Chaotic with mode 4 to mode 8 of IEEE 802.11n PHY and simulated the BLER performance over AWGN channels and multipath fading channels. Massive simulation results demonstrate that HARQ-Chaotic has about $1-4 d B$ gain comparing with traditional HARQ-CC.

\section{Appendix}

Suppose the source symbol vector is $\overrightarrow{\boldsymbol{x}}$, and $x$ is an arbitrary symbol of this vector. After analog chaotic encoding with coding rate of $1 / 2$, we get codewords of $\{x[0], x[1]\}$, where

$$
\begin{aligned}
& x[0]=x \\
& x[1]= \begin{cases}-2 x[0]+0.5, & 0<x[0]_{i} \leq 0.5 \\
2 x[0]+0.5, & -0.5 \leq x[0]_{i} \leq 0\end{cases}
\end{aligned}
$$

After AWGN channel, the receiver receives 2 codewords of $\{y[0], y[1]\}$ with Gaussian noise,

$$
\begin{aligned}
& y[0]=x[0]+n_{0} \\
& y[1]=x[1]+n_{1}
\end{aligned}
$$

where $n_{0}$ and $n_{1}$ are random Gaussian noises with zero mean and variance (or power) of $\sigma^{2}$.

After decoding, we can get the estimated source symbol in the correct interval as follows:

$$
\begin{aligned}
& \tilde{x}=\frac{y[0]+2 y[1]-1}{5}=x+\frac{n_{0}}{5}+\frac{2 \times n_{1}}{5}, \\
&-0.5 \leq \tilde{x} \leq 0 \\
& \tilde{x}=\frac{y[0]-2 y[1]+1}{5}=x+\frac{n_{0}}{5}-\frac{2 \times n_{1}}{5}, \\
& 0<\tilde{x} \leq 0.5 .
\end{aligned}
$$

So the additive noise of the estimated source becomes $n_{0} / 5+\left(2 \times n_{1}\right) / 5,-0.5 \leq x \leq 0$ or $n_{0} / 5-\left(2 \times n_{1}\right) / 5,0<$ $x \leq 0.5$. These two kinds of noise are still belonging to Gauss distribution, and the powers of them are both $\sigma^{2} / 5$.

\section{Data Availability}

The data used to support the findings of this study are available from the author Wei Yu (2014yuwei@tongji.edu.cn) upon request.

\section{Conflicts of Interest}

The authors declare that they have no conflicts of interest.

\section{Authors' Contributions}

Wei Yu contributed to the simulation program and part of the writing of this paper. Fusheng ZHU contributed to the theoretical analysis and revision work after reviewing of this paper. Jun Wu contributed to the conceiving, framework, and part of the writing of this paper. Rui Wang, Zhifeng Zhang, and Haoqi Ren contributed to the guidance of the simulation program and the revision work before submission of this paper. 


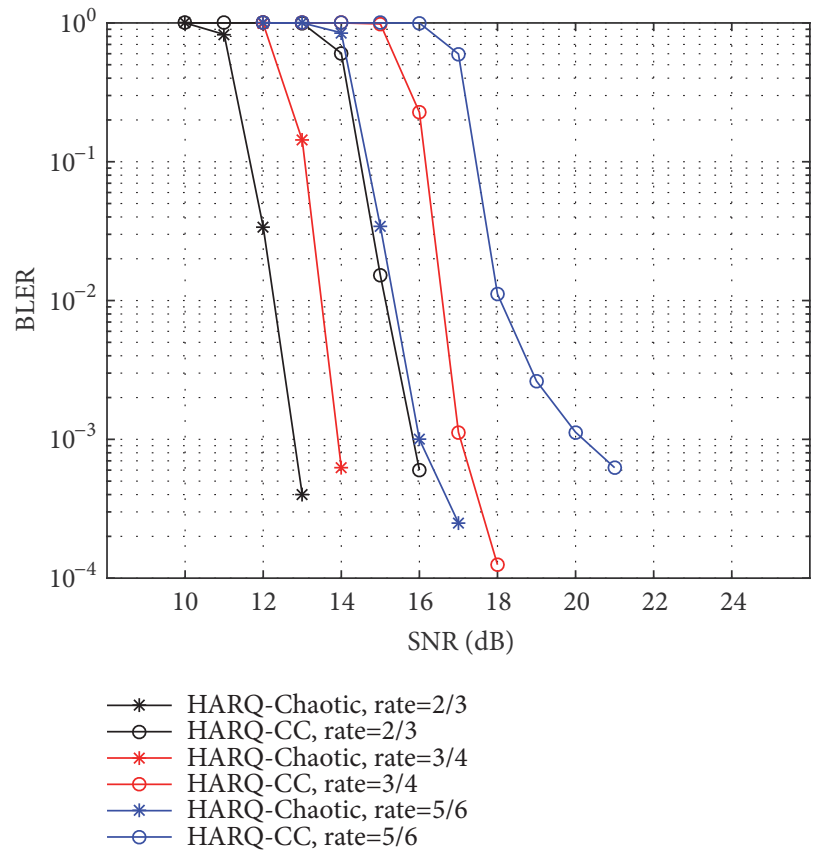

FIGURE 12: BLER of 2 retransmissions with LDPC coding and 64-QAM modulation in fading channels.

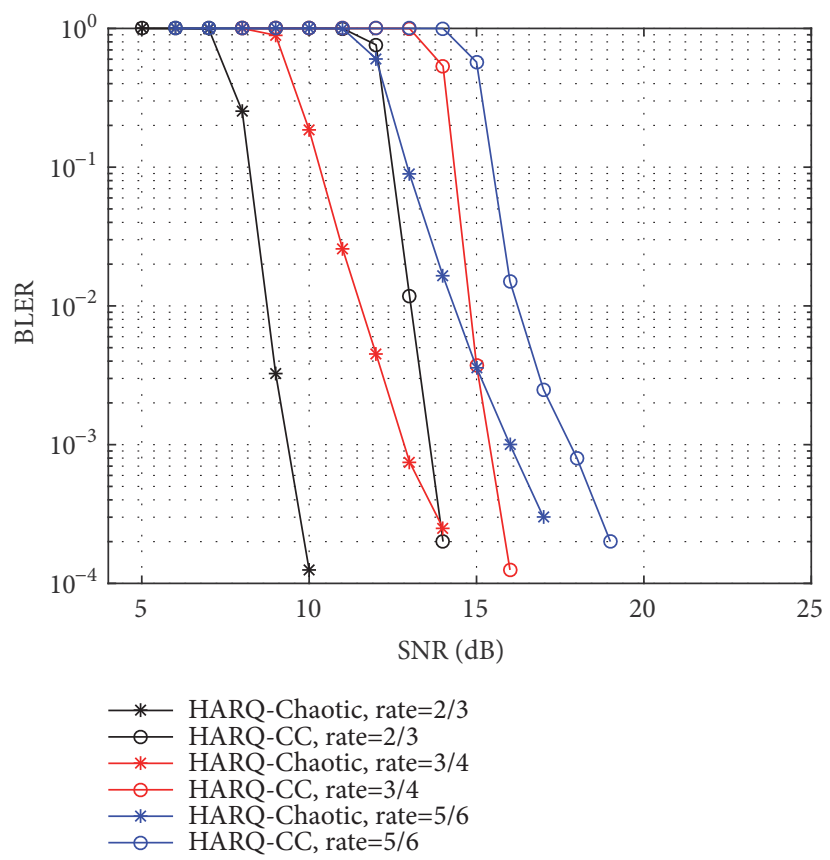

FIGURE 13: BLER of 3 retransmissions with LDPC coding and 64-QAM modulation in fading channels.

\section{Acknowledgments}

This work was supported in part by the National Science Foundation China under Grant 61571329, Grant 61831018, and Grant 61631017 and Guangdong Province Key Research and Development Program Major Science and Technology Projects under Grant 2018B010115002.

\section{References}

[1] H. Cui, C. Luo, K. Tan, F. Wu, and C. W. Chen, "Seamless rate adaptation for wireless networking," in Proceedings of the Seamless Rate Adaptation for Wireless Networking, MSWiM, pp. 437-446, October 2011.

[2] S. Sesia, I. Toufik, and M. Baker, LTE - The UMTS Long Term Evolution: From Theory to Practice, Wiley, 2009. 
[3] J. Andrews, A. Ghosh, and R. Muhamed, Fundamentals of WiMAX, Prentice Hall, 2007.

[4] EEE Std 802.16-2004, IEEE standard for local and metropolitan area networks, Part 16: air interface for fixed broadband wireless access systems, Oct 2004.

[5] IEEE Std 802.16e-2005, IEEE Standard for Local And Metropolitan Area Networks, Part 16: Air Interface for Fixed Broadband Wireless Access Systems, Amendment 2: Physical And Medium Access Control Layers for Combined Fixed And Mobile Operation in Licensed Band, Feb 2006.

[6] IEEE 802.11n, Wireless LAN medium access control (MAC) and physical layer (PHY) specifications: amendment 5 - enhancements for higher throughput, Sept 2007.

[7] D. Chase, "A combined coding and modulation approach for communication over dispersive channels," IEEE Transactions on Communications, vol. 21, no. 3, pp. 159-174, 1973.

[8] S. Lin, D. J. Costello, and M. J. Miller, "Automatic-RepeatRequest Error Control Schemes," IEEE Communications Magazine, vol. 22, no. 12, pp. 5-17, 1984.

[9] D. Toumpakaris, J. Lee, A. Matache, and H.-L. Lou, "Performance of MIMO HARQ under receiver complexity constraints," in Proceedings of the 2008 IEEE Global Telecommunications Conference, GLOBECOM 2008, pp. 1-5, Nov 2008.

[10] K. Xie, J. Li, and Y. Liu, "Linear analog codes: The good and the bad," in Proceedings of the 2012 46th Annual Conference on Information Sciences and Systems (CISS), pp. 1-6, March 2012.

[11] Y. Liu, J. Li, and K. Xie, "Analysis of linear channel codes with continuous code space," in Proceedings of the 2012 46th Annual Conference on Information Sciences and Systems, CISS 2012, pp. 1-6, March 2012.

[12] B. Chen and G. W. Wornell, "Analog error-correcting codes based on chaotic dynamical systems," IEEE Transactions on Communications, vol. 46, no. 7, pp. 881-890, 1998.

[13] K. Xie and L. Li (Tiffany), "Chaotic analog error correction codes: The mirrored baker's codes," in Proceedings of the GLOBECOM 2010 - 2010 IEEE Global Communications Conference, pp. 1-5, December 2010.

[14] G. Kaddoum, "Wireless chaos-based communication systems: a comprehensive survey," IEEE Access, vol. 4, pp. 2621-2648, 2016.

[15] V. Lynnyk and S. Celikovský, "On the anti-synchronization detection for the generalized Lorenz system and its applications to secure encryption," Kybernetika, vol. 46, no. 1, pp. 1-18, 2010.

[16] M. Herceg, G. Kaddoum, D. Vranjes, and E. Soujeri, "Permutation index DCSK modulation technique for secure multiuser high-data-rate communication systems," IEEE Transactions on Vehicular Technology, vol. 67, no. 4, pp. 2997-3007, 2018.

[17] G. Kolumban, M. P. Kennedy, and L. O. Chua, "The role of synchronization in digital communications using chaos. II. Chaotic modulation and chaotic synchronization," IEEE Transactions on Circuits and Systems I: Fundamental Theory and Applications, vol. 45, no. 11, pp. 1129-1140, 1998.

[18] G. Kaddoum, F.-D. Richardson, and F. Gagnon, "Design and analysis of a multi-carrier differential chaos shift keying communication system," IEEE Transactions on Communications, vol. 61, no. 8, pp. 3281-3291, 2013.

[19] X. Min, W. Xu, L. Wang, and G. Chen, "Promising performance of a frequency-modulated differential chaos shift keying ultrawideband system under indoor environments," IET Communications, vol. 4, no. 2, pp. 125-134, 2010.

[20] H. Yang, W. K. S. Tang, G. Chen, and G.-P. Jiang, "System Design and Performance Analysis of Orthogonal Multi-Level
Differential Chaos Shift Keying Modulation Scheme," IEEE Transactions on Circuits and Systems I: Regular Papers, vol. 63, no. 1, pp. 146-156, 2016.

[21] H. C. Papadopoulos and G. W. Wornell, "Maximum-Likelihood Estimation of a Class of Chaotic Signals," IEEE Transactions on Information Theory, vol. 41, no. 1, pp. 312-317, 1995.

[22] C. Pantaleon, L. Vielva, D. Luengo, and I. Santamaria, "Estimation of a certain class of chaotic signals: an em-based approach," in Proceedings of the IEEE International Conference on Acoustics, Speech, and Signal Processing (ICASSP), vol. 2, pp. 1129-1132.

[23] C. Pantaleón, L. Vielva, D. Luengo, and I. Santamaría, "Bayesian estimation of chaotic signals generated by piecewise-linear maps," Signal Processing, vol. 83, no. 3, pp. 659-664, 2003.

[24] C. Pantaleon, D. Luengo, and I. Santamaria, "Bayesian estimation of a class of chaotic signals," in Proceedings of the 2000 International Conference on Acoustics, Speech and Signal Processing, vol. 1, pp. 193-196, Istanbul, Turkey.

[25] K. Xie, P. Tan, N. B. Chong, and J. Li, "Analog turbo codes: A chaotic construction," in Proceedings of the IEEE International Symposium on Information Theory, 2009, pp. 894-898, 2009.

[26] Y. Liu, J. Li, and K. Xie, "Efficient image transmission through analog error correction," in Proceedings of the 2011 IEEE 13th International Workshop on Multimedia Signal Processing (MMSP), pp. 1-6, Oct 2011.

[27] Y. Liu, J. Li, X. Lu, C. Yuen, and J. Wu, "A family of chaotic pure analog coding schemes based on baker's map function," EURASIP Journal on Advances in Signal Processing, vol. 2015, no. 1, pp. 1-18, 2015.

[28] B. Tan, J. Wu, Y. Li, H. Cui, W. Yu, and C. W. Chen, "Analog Coded SoftCast: A Network Slice Design for Multimedia Broadcast/Multicast," IEEE Transactions on Multimedia, vol. 19, no. 10, pp. 2293-2306, 2017.

[29] W. Yu, J. Wu, Y. Li, and B. Zhang, "A hybrid digital-analog chaotic code," IEEE Wireless Communications Letters, 2018.

[30] J. Perry, P. A. Iannucci, K. E. Fleming, H. Balakrishnan, and D. Shah, "Spinal codes," in Proceedings of the ACM SIGCOMM Conference Applications, Technologies, Architectures, and Protocols for Computer Communication, SIGCOMM '12, pp. 49-60, August 2012.

[31] Z. Zhao, L. Zhou, X. Zheng, and S.-E. Park, "Enhanced constellation rearrangement for HARQ with symbol combining," in Proceedings of the 2009 IEEE Mobile WiMAX Symposium, MWS 2009, pp. 11-15, USA, July 2009.

[32] E. W. Jang, J. Lee, L. Song, and J. M. Cioffi, "An efficient symbollevel combining scheme for MIMO systems with hybrid ARQ," IEEE Transactions on Wireless Communications, vol. 8, no. 5, pp. 2443-2451, 2009.

[33] E. W. Jang, J. Lee, H.-L. Lou, and J. M. Cioffi, "On the combining schemes for MIMO systems with hybrid ARQ," IEEE Transactions on Wireless Communications, vol. 8, no. 2, pp. 836842, 2009.

[34] S. Park and S. Choi, "Performance of Symbol-Level combining and Bit-Level combining in MIMO multiple ARQ systems," IEEE Transactions on Communications, vol. 64, no. 4, pp. 15171528, 2016.

[35] M.-S. Alouini and A. J. Goldsmith, "Adaptive modulation over Nakagami fading channels," Wireless Personal Communications, vol. 13, no. 1, pp. 119-143, 2000. 
[36] D. L. Goeckel, "Adaptive coding for time-varying channels using outdated fading estimates," IEEE Transactions on Communications, vol. 47, no. 6, pp. 844-855, 1999.

[37] D. Qiao, S. Choi, and K. G. Shin, "Goodput analysis and link adaptation for IEEE 802.11 a wireless LANs," IEEE Transactions on Mobile Computing, vol. 1, no. 4, pp. 278-292, 2002.

[38] J. Medbo, H. Hallenberg, and J. Berg, "Propagation characteristics at $5 \mathrm{ghz}$ in typical radio-lan scenarios," in Proceedings of the IEEE 49th Vehicular Technology Conference, vol. 1, pp. 185-189, September 1999.

[39] J. Medbo and P. Schramm, Channel models for hiperlan/2 1998. ETSI/BRAN doc, (3ERI085B). 


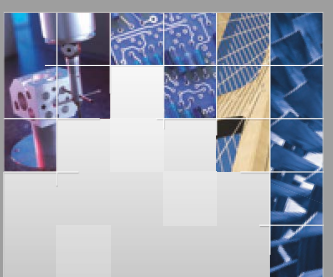

\section{Enfincering}
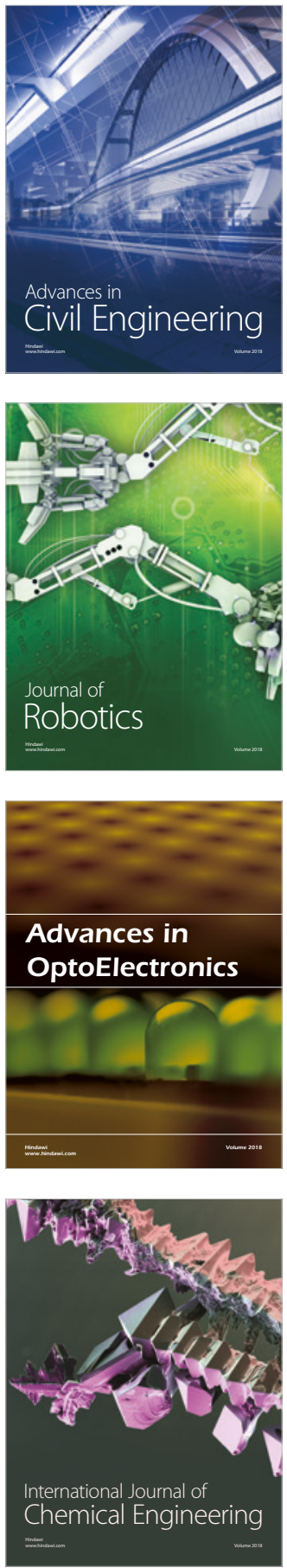

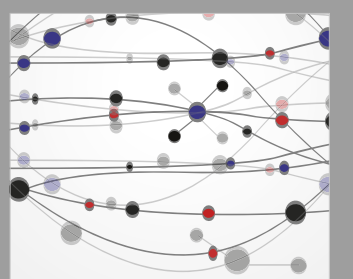

\section{Rotating \\ Machinery}

The Scientific World Journal

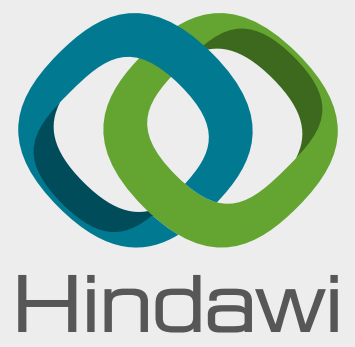

Submit your manuscripts at

www.hindawi.com
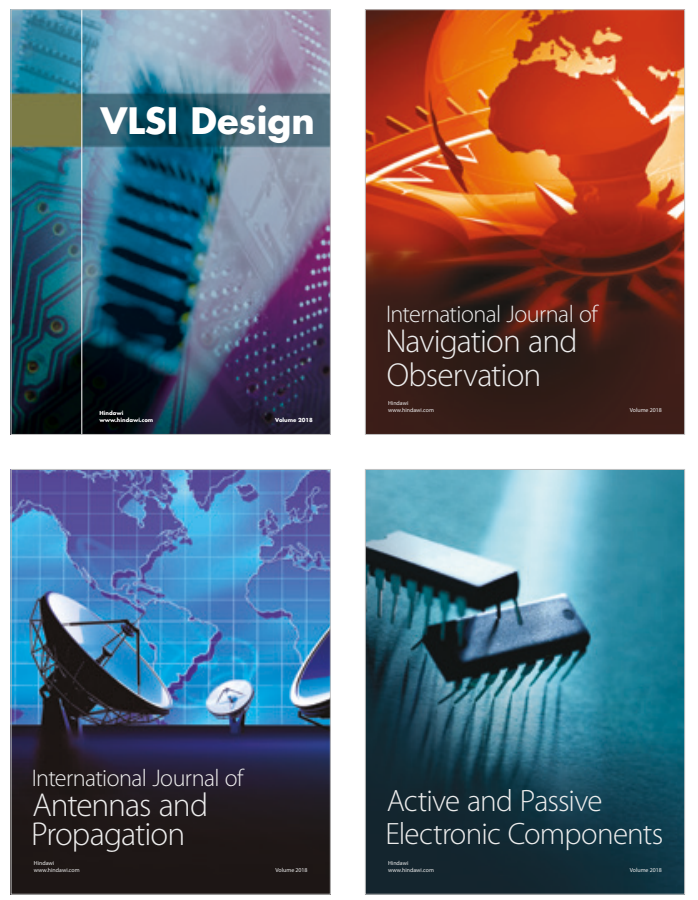
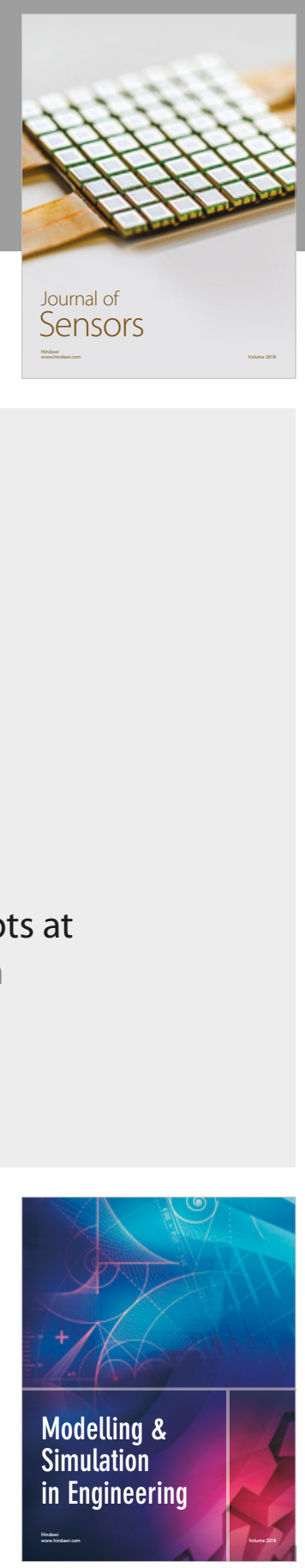

\section{Advances \\ Multimedia}
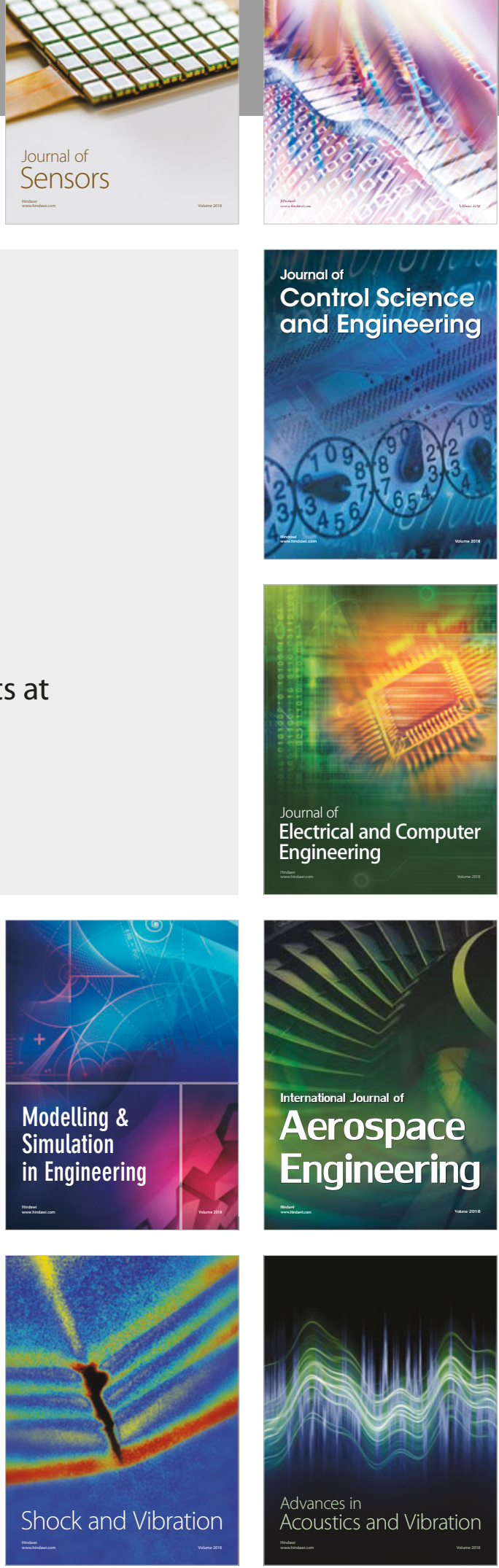